Sociedades Precapitalistas, vol. 6, nº 2, e017, junio 2017. ISSN 2250-5121

Universidad Nacional de La Plata.

Facultad de Humanidades y Ciencias de la Educación.

Centro de Estudios de Sociedades Precapitalistas (CESP)

\title{
kkw y kkw-zmAw. Un estudio del concepto de oscuridad en la Duat de los Libros del Más Allá del Reino Nuevo Egipcio1
}

\author{
kkw y kkw-zmAw. A survey about darkness concept through the \\ Duat of the Egyptian New Kingdom Books of the Underworld
}

\section{Mariano Bonanno *}

* Instituto de Historia Antigua Oriental "Dr. Abraham Rosenvasser" Facultad de Filosofía y Letras - Universidad de Buenos Aires, Argentinal mbonanno1971@gmail.com

\section{PALABRAS CLAVE RESUMEN}

Duat Los Libros del Más Allá del Reino Nuevo hablan incesantemente del estado pre y post paso de Ra por la Duat. El contexto de oscuridad revierte en luz, calor y movimiento con su presencia, para los difuntos que en la tierra actuaron conforme a mAat; los condenados, por el contrario, sufrían las puniciones consecuencia de su iniquidad. Los egipcios disponían de varios vocablos para aludir a la idea de oscuridad, pero sin lugar a dudas kkw y kkw-zmAw son los que más controversia nos generan dada la falta de límites y las eventuales superposiciones que entre ambas acepciones se producen. El presente artículo propone, además de profundizar los pioneros estudios de Hornung (1956a y 1956b), revisar ambos conceptos, contextualizarlos a los libros funerarios del Reino Nuevo -Libro del Amduat, Libro de las Puertas, Libro de las Cavernas y Libro de la Tierra-, y poner en consideración las definiciones que taxativamente proponen espacios y áreas de influencia precisas.

\section{KEYWORDS ABSTRACT}

Duat The New Kingdom Books of the Underworld express incessantly status pre and post transit about Ra through Duat. The context of darkness reverses in light, warm and motion with its presence for the Darkness deceased who on earth acted according mAat; the damned, by contrast, suffered the punishment because of their iniquity. Egyptians possessed various words to designate to the idea of darkness but undoubtedly kkw and kkw-zmAw which are the terms that more controversy generates given the lack of limits and eventual overlaps occurring between both meanings. This article proposes, in addition to deepening on the pioneering Hornung's studies (1956a and 1956b), review both concepts contextualizing them to the New Kingdom funerary literature -Book of Amduat, Book of Gates, Book of Caverns, and Book of the Earth-, and put into consideration the definitions that specifically spaces and precise areas of influence suggest. 


\section{1- kkw-zmAw como espacio fuera de la creación. Literatura funeraria y cosmología}

La connotación que habitualmente se le ha dado a kkw-zmAw como espacio por fuera del mundo ordenado o límite último -espacial y temporal- de la creación $\underline{2}$, es el resultado, o mejor dicho, surge a partir de ciertas alusiones de carácter cosmológico, tal como la que surge de la denominada "cosmología de Seti I y Rameses IV” (Parker, 1974: 55; Neugebeuer y Parker, 1960), o también llamada cosmología o Libro de Nut (Von Lieven, 2007 y 2010).

Las copias más tempranas de estos textos se hallan en el cenotafio de Seti I (Murray, 1904; Frankfort, 1933), y en la cámara del sarcófago de la tumba de Rameses IV (Hornung, 1990). Estos textos fueron copiados más tarde, con comentario en demótico en un papiro del siglo II B.C.E. en el Fayum -uno de los pocos ejemplos sobrevivientes de la discusión de los egipcios de sus propios textos filosóficos- (Allen, 1988:1). Este papiro es el célebre Papiro Carlsberg (Von Lieven, 2007: 9-13) (c. 144 CE) ${ }^{3}$ en sus dos versiones (pCarlsberg1, PC1) que trata sobre el denominado Libro de Nut, y una versión abreviada o fragmentaria, el llamado Papiro Carlsberg 1a (pCarlsberg1a, PC1a) que probablemente, se presume, ha sido escrita por el mismo escriba (Symons, 1999:155) que PC1. En ambos, de entre una miscelánea de textos médicos, se explicitan estas mismas alusiones mitológicas ${ }^{4}$.

Como el lado superior de este cielo que está en oscuridad uniforme

[kkw-zmAw]

Los límites del sur, norte, oeste y este son desconocidos,

Habiendo sido fijados en el Abismo, en la inacción $\underline{\underline{5}}$,

No hay luz allí del carnero [bA de Ra]: él no aparece allí...

un lugar cuya tierra sur, norte, oeste y este es desconocida para los

dioses o Ax.w,

No existiendo allí claridad. Cada lugar está vacío de cielo y vacío de

tierra.

Esta es la completa Duat $\underline{\underline{6}}$.

El texto alude a un espacio amorfo, desconocido, ilimitado, inaccesible que es asimilado a la Duat, espacio de los difuntos. La importancia de la cita es clara puesto que establece la composición y ubicación de uno de los componentes más controversiales de la cosmología egipcia. En este caso, la indefinición que supone esta oscuridad uniforme=abismo primordial (acuoso) sugerido por Allen ${ }^{\underline{7}}$, nos ubica en un espacio incierto, inerme, en cuyo interior los límites son vagos e ininteligibles, y finalmente espacio sin luz y desconocido por dioses y Ax.w. La uniformidad de esta oscuridad contenida en el concepto zmAw (uniforme, completa, absoluta), tal como veremos más abajo, marca ya una primera aproximación a lo que la construcción kkwzmAw significa per se y al concepto de kkw, la otra denominación para oscuridad.

En un contexto cosmológico como el aquí propuesto, la delimitación espacial se nos presenta evidente, al punto que puede esbozarse una configuración del universo egipcio incluyendo estos espacios caóticos sin perjuicio de ocasionales superposiciones, indiferenciaciones $\stackrel{8}{\text {. }}$

El problema surge cuando estos espacios vinculados al caos aparecen en otro contexto como en el caso específico de la literatura funeraria, motivo por el cual las anteriores definiciones comienzan a aparecer como indiferenciadas y a interactuar con otras realidades.

Retomando el carácter cosmológico del texto del Libro de Nut, focalizaremos en lo que supone el concepto de kkw zmAw enmarcado en un "concepto del universo" - y como tal, configurando una totalidad (ordenada y no ordenada), e inmerso en (y con) otras realidades y espacios en un marco de referencia dinámico e interactivo. En otras palabras, hay una evidente intencionalidad a la hora de enmarcar un concepto en un 
ámbito cosmológico que se difumina, como a continuación veremos, en el conjunto amplio de literatura funeraria.

En el caso particular de la literatura funeraria, debemos considerar la vinculación de la dinámica que los textos imprimen a la Duat no sólo como ámbito de metamorfosis solar, sino también como vehículo diario de movimientos, respiraciones, efímeros despertares y ejecuciones, con la idea de inmutabilidad consubstancial al pensamiento religioso egipcio.

Podemos asumir entonces que dinámica y estática, la simultaneidad y la sucesión de los acontecimientos, la espacialidad y la temporalidad se proyectan conjuntamente en la completa composición del Amduat (Hegenbarth-Reichardt, 2009:13).

Así, la dinámica del tiempo en la Duat reviste una secuencialidad, en lo que se refiere a la generalidad de sus habitantes $\frac{10}{}$, pero también una simultaneidad en lo que concierne a Ra, consecuencia de su dominio de la temporalidad. En consecuencia, la conformación y variabilidad antropológica de justificados y condenados se sujeta a una secuencia que se inicia en la objetivación y culmina con la vuelta al estado pre-objetivado, es decir, un retorno a la realidad previa a la presencia solar.

De este modo, es preciso circunscribir el locus de los diferentes espacios que conlleva la precisión propia de textos de carácter cosmológico (descriptivos) en los que, aun a pesar de eventuales contrastes, o incluso ambivalencias, se concluye en una narración que delimita taxativamente procesos pero sobre todo ámbitos $\underline{11}$.

Las diferencias en el análisis de un espacio en un texto cosmológico y en un texto funerario es patente en la referencia de Conman (2003:26), en la que confirma que el papiro Carlsberg I. D. 11.32 dice que las tierras de la dwAt (el punto en el cual el sol es rejuvenecido a sí mismo en la noche) están en las alturas. La línea siguiente (11.33) refiere que las tierras de la dwAt están vacías. La comparación de este "vacío" de la Duat con su superpoblación en los textos funerarios del Reino Nuevo es inevitable. No debemos olvidar que en la Duat del Reino Nuevo, la Duat supone multiplicidad y espacialidad, siendo una colosal extensión inmersa en una compleja trama de jerarquías que oscilan entre divinidades tutelares, faraón, plétora de dioses, hasta colaboradores, genios, barqueros, etc. Ello como consecuencia de la ampliación genera del espacio, consecuencia a su vez de la posibilidad para todos los difuntos de compartir las vicisitudes del paso del disco, otrora privativo del faraón o sus allegados $\underline{12}$.

Es en la dinámica de la Duat, en la que las “entradas y salidas” del disco en el paisaje general son constantes y lo delinean permanentemente, en la que la presencia de kkw-zmAw en la primera y en la última hora de la noche reflejan una diversidad que excede a esta acotada definición y pone en duda la presunta ubicación de kkw-zmAw por fuera del mundo ordenado.

Siguiendo este argumento, uno se pregunta de qué modo un espacio que está por fuera del mundo ordenado -kkw-zmAw-, puede participar activamente en éste en un marco general de activación y dinamismo permanentes $\frac{13}{}$. Ello es lo que intentaremos dilucidar en las páginas siguientes, juntamente con el análisis de lo que el concepto general de oscuridad supone en la dinámica inherente a la Duat.

\section{2 - kkw y kkw-zmAw como inflexiones en la Duat}

Es conveniente destacar que en las citas textuales referidas a lo largo del texto se optará por las denominaciones respectivas de kkw o kkw-zmAw, manteniendo el original egipcio, por sobre oscuridad, oscuridad absoluta, oscuridad completa, tinieblas de conexión, oscuridad unificada, tinieblas primordiales, etc..., ello como consecuencia del modo difuso y flexible que ambos términos aparecen en las fuentes.

Si existe una constante en el paisaje de la Duat, seguramente la oscuridad, o la penumbra o las tinieblas sean 
la marca. Como espacio oscuro, con matices según el área específica $\underline{14}$, la oscuridad es la realidad a superar, abriéndola, para luego pasarla y finalmente volver a ella. Es precisamente ésta la dinámica circular de la Duat, oscuridad-luz-oscuridad o con énfasis en el desplazamiento del disco, quietud-movimientos-quietud $\underline{15}$.

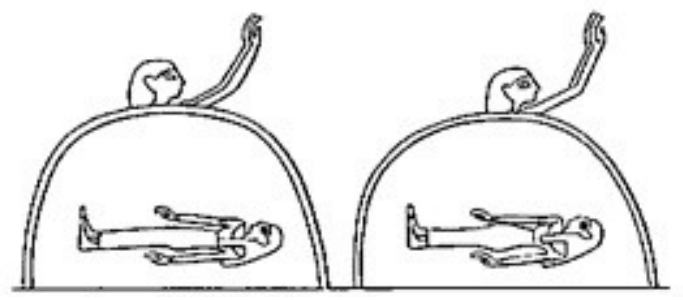

Imagen $\mathrm{N}^{\circ} 1$ : Efectos regenerativos (dinámica) del paso solar

(Libro de la Tierra, A,2,5) Piankoff (1954:Fig. 96)

En esta imagen se observa la secuencia en la que las “momias en sus montículos” son sometidas al paso del disco. A la quietud / oscuridad del estado propio de los habitantes de la Duat, el disco les insufla luz, calor y movimientos con su presencia. De este modo, en este contexto, la circularidad opera en forma manifiesta tanto en la anulación provisoria de la oscuridad como en la activación, también provisoria, del movimiento. La provisoriedad se sujeta, pues, a la secuencia que supone la ausencia / presencia / ausencia.

Como oscuridad o "tinieblas del crepúsculo" (Piankoff, 1942a:7), como "oscuridad completa” (Roberson, 2007:104), como "oscuridad unificada” (Abt \& Hornung, 2007:.357), "tinieblas primordiales” (Hornung, 1975b:1153 y 1999a:164), como "tinieblas condensadas” (Morenz, 1992:168), como "tinieblas unificadas” (Manassa, 2006, p.132), o como tinieblas de conexión (Hornung, 1975b:1153 y 1956b: 30), "oscuridad uniforme” (Allen, 1988, p.6), “densa oscuridad” (Murray, 1904:21), “oscuridad concentrada” (Hornung, 1956a:33), kkw-zmAw corresponde, o a la zona de contacto con el crepúsculo, o bien con el amanecer $\frac{16}{y}$ al igual que el nwn “corresponde a la ubicuidad del mundo de los muertos” (Hornung, 1956b:30) ${ }^{17}$,

1) "Él (la serpiente "la que vive en la tierra") se levanta contra kkw-zmAw...Es la serpiente protectora de la Duat, el bA sagrado -Dsrt- del Primero de los Occidentales”.

2) "Son ellos (doce dioses con armas delante de la serpiente) los que rechazan a la "Cara maligna” -nHA-Hr- en kkw-zmAw (Libro del Amduat, 10,2)

Del mismo modo, kkw-zmAw se corresponde también con el extremo o límite del mundo ordenado;

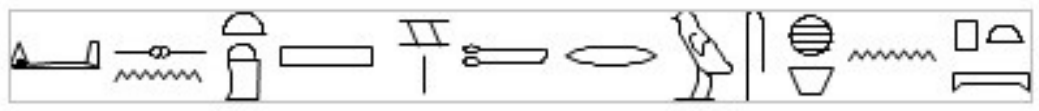

rdj=sn tAS=T r wsx n pt

"se les dio tu frontera hasta el ancho del cielo

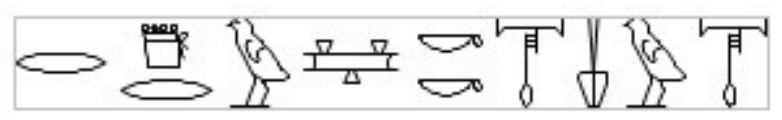

r Drw kkw-zmAw

(hacia) el final de kkw-zmAw 


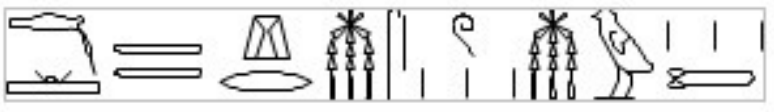

$\mathrm{mH}$ tAwy Xr msw msw=T $\underline{18}$

(para) llenar las Dos Tierras debajo de los nacidos de tus nacimientos”

Para Hornung, kkw-zmAw es parte del Nun (Hornung, 1956a:32-33) $\underline{19}$ y debería traducirse o como “vereinigte Finsternis” (Hornung, 1975b:1153), o también como “zusammengeballte Finsternis” (Hornung, 1956b:30), tinieblas de conexión o unificadas (ver p. 4). Siguiendo la argumentación, en el Wb (Wb V: 143144) se afirma que la expresión corresponde a "el alba y el crepúsculo en los límites de la Duat en la primera y en la última hora de la noche”; es decir, la oscuridad iluminada al alba y oscuridad o sombra al anochecer, conclusión que es rechazada acertadamente por Hornung por inexacta e insuficiente.

En este sentido, además de ser parte de la oscuridad previa y posterior a la entrada y salida del disco en la Duat, puede entenderse además como la oscuridad que acompaña a sus habitantes en ausencia de Ra. Por esta razón, la definición de Hornung como "tinieblas unificadas” nos parece pertinente no sólo lingüística, sino también operativamente, junto con la definición de "primordiales” o “absoluta”.

En cuanto a la oscuridad en sentido amplio, no habría motivos para diferenciar kkw de kkw-zmAw, y a éste último con la Duat -Unterwelt- (Barta, 1985:179, nota 735)ํㅡㄹ Wb, “oscuridad del anochecer y del alba”, no podríamos explicar, por ejemplo, el porqué de la aparición de kkw-zmAw en las dos oportunidades anteriores (Libro del Amduat, 10,2).

Por el contrario, kkw daría marco general a la oscuridad habitual que rodea a los bAw que forman parte del séquito de Ra, y como tales, receptores de las objetivaciones diarias; desde este lugar podría hacerse una analogía entre kkw y grH (Finsternis/Nacht) (Weibach-Koepke, 2007:50) asumiendo un mero contexto de oscuridad sin ninguna connotación adicional. De este modo, las grafías para kkw serían las siguientes:
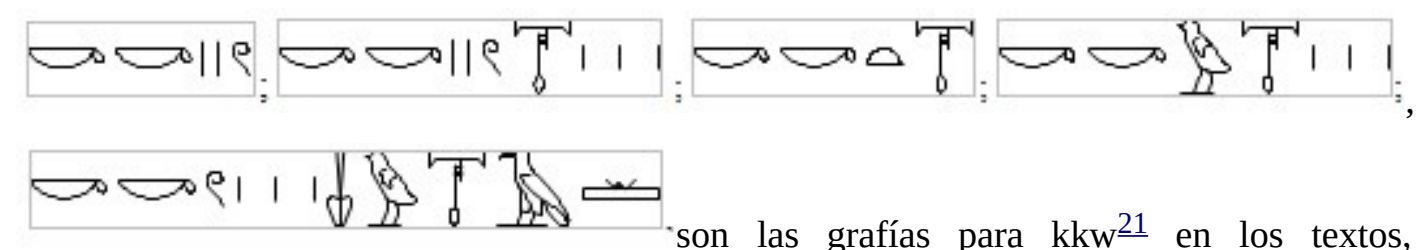

son las grafías para $\mathrm{kkw}^{21}$ en los textos, oscilando entre traducciones habituales como "oscuridad”, “penumbras” o "tinieblas” sin ninguna otra connotación tal como kkw- zmAw.

Para kkw- zmAw las opciones son aún mayores; de este modo puede encontrarse

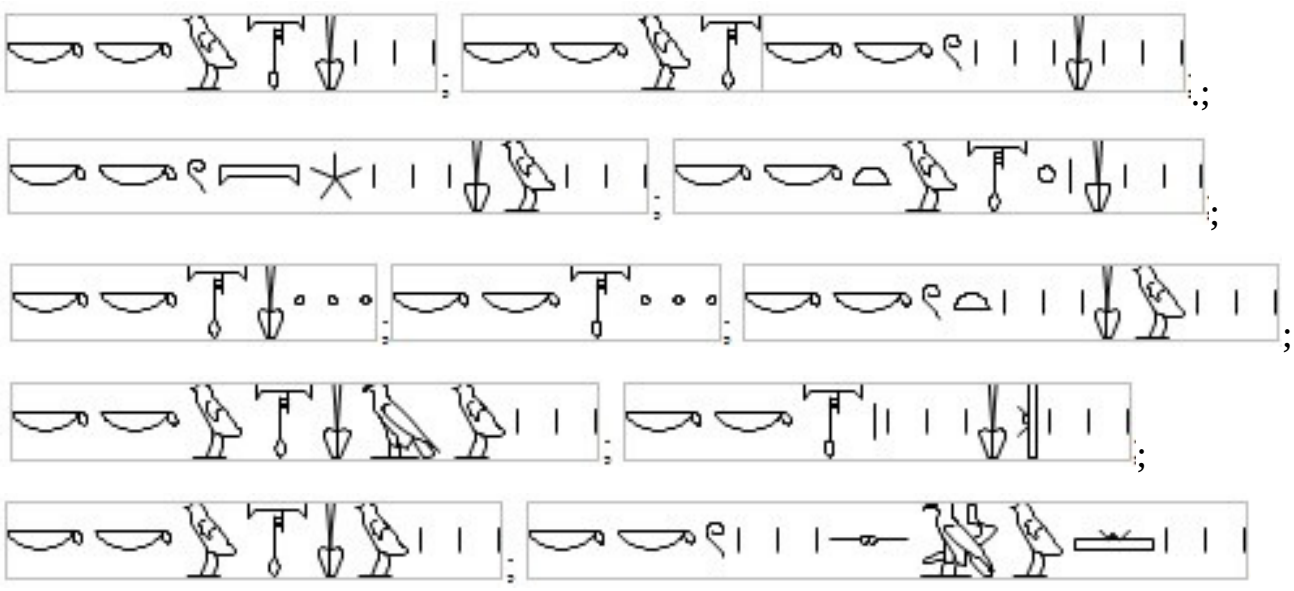

Un dato importante, y que puede marcar una de las diferencias entre ambas acepciones tiene vinculación con 
las acciones que son ejercidas sobre una, kkw, por sobre la otra, kkw- zmAw. Los textos nos refieren que el disco de la Duat descansa, pasa, ilumina, habla, vuelve, entra, penetra en kkw, mientras que en las alusiones a kkw- zmAw las acciones son más restringidas y tienen que ver principalmente con entrar e iluminar. La oscuridad disipada o la oscuridad que retorna luego de la presencia de la luz del disco es kkw $\underline{22}$.

Un par de referencias interesantes a la caverna de Osiris en el Libro de las Cavernas aluden a su oscuridad absoluta -kkw-zmAw- $(5,3,1)^{\underline{23}}$ y a su oscuridad -kkw- $(5,5,1)^{\underline{24}}$, lo que podría significar una alternancia indiferenciada en el uso del concepto. No es esta la única ocasión en que ello sucede, pero evidentemente debe haber una razón de carácter o funcional, o denotativa en lo que ambas acepciones suponen.

Probablemente, la utilización de kkw-zmAw sobre kkw obedezca a la hipérbole que demanda el contexto particular cuando se involucra al bA de Osiris $\underline{25}$, o al espacio en el que se oculta el enemigo del sol -Apep- en cualquiera de sus denominaciones, o bien cuando se referencia a Ra. La siguiente cita ilustra la diferencia entre la oscuridad que involucra a los habitantes en general $\frac{26}{y}$ aquella que la actividad de Ra en este caso demanda.

Son ellos [habitantes del registro] quienes hacen que los bAw consigan dormir. Esto es lo que hacen: hacen que venga kkw, derrotan a los enemigos en su hora. Son ellos los que mantienen la protección durante el día, traen la noche para que el gran dios pueda establecer kkw-zmAw, para descansar en la puerta del horizonte oriental del Cielo. (Libro del Amduat, 2, 1)

Tengamos en cuenta que estamos frente a textos funerarios que conforman una organicidad cósmica necesaria para la continuidad de Egipto, y como tales, contralor a los efectos de la disgregación y una posibilidad tangible para quienes habitan la Duat de retorno a la plenitud ontológica que la muerte interrumpió.

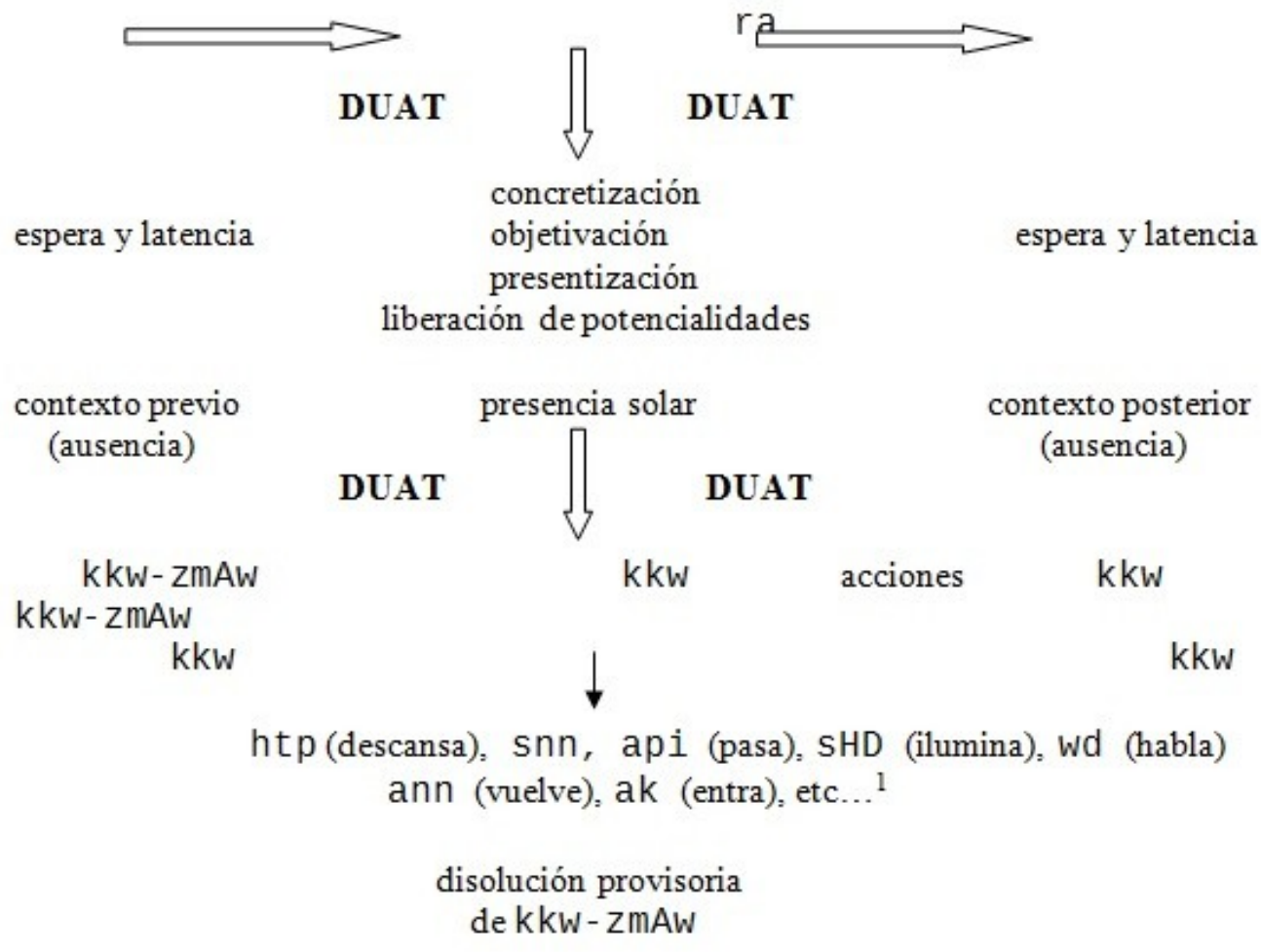

${ }^{1}$ Para las acciones en la Duat, ver Wiebach-Koepke, (1997, pp.341-347). 
Las aperturas de la Duat, a juzgar por la significación que sobre sus habitantes conllevan, suponen una recepción entusiasta como expectante para el ingreso solar, y un alejamiento signado por el desánimo que genera una nueva espera de restauración $\underline{27}$.

La connotación de un contacto entre el mundo de los muertos -espacio transformador y regenerativo- y el de los vivos, es una transición de crisis cósmica en la que los límites se difuminan para coincidir en la inflexión de la incertidumbre y la restitución de la continuidad. Esta dilución de espacios, realidades y estados conectados, exige la interpenetración por cuanto la re-edición del orden remite al caos, a la indefinición, portadores naturales de taumaturgia cósmica.

La majestad de este Gran Dios descansa en esta caverna en el final de kkw-zmAw. Nacido será este Gran Dios en sus formas [xprw] de Jepri en esta caverna. Nun y Nunet, Heh y Hehet aparecen [xpr] en esta caverna en el nacimiento de este Gran Dios para que él pueda salir de la Duat, bajar en la Barca de Día, y salir de los muslos de Nut. (Libro del Amduat, 12, Int.)

En esta cita del Libro del Amduat, Ra renace como Khepri de los muslos de Nut abandonando kkw-zmAw de la Duat que es descrita como un abismo y como abrazada por Shu. La mención de nwn, nwnt y HH, HHt incorpora al proceso solar dos parejas de dioses primordiales de la teología de Hermópolis, representando respectivamente al Abismo y a la Inundación, figuras prototípicas del recorrido de $\mathrm{Ra} \underline{28}$.

En el Libro de las Puertas, Ra abandona el Mundo de Occidente y descansa definitivamente en el cuerpo de Nut. Los pilares de la puerta de esta división nos muestran a Khepri como el sol en su devenir y Atum como aquel que diviniza a Ra, papel que ya cumplía desde comienzos del viaje, al "espiritualizarlo”르. Veamos a continuación las citas de los dos vocablos en los textos aquí estudiados:

\begin{tabular}{|c|c|c|c|}
\hline \multicolumn{2}{|c|}{ Libro del Amduat } & \multicolumn{2}{|c|}{ Libro de las Puertas } \\
\hline kkw & kkw zmAw & kkw & kkw zmAw \\
\hline $2,3(2)$ & 2,1 & 2,2 & $1^{\circ}$ Puerta \\
\hline 3,3 & 2,2 & $1^{\circ}$ Puerta & $2^{\circ}$ Puerta \\
\hline $6,1(3)$ & 2,3 & $3^{\circ}$ Puerta & $4^{\circ}$ Puerta \\
\hline 9,1 & 5,3 & $3,1 \mathrm{snk}$ & $5^{\circ}$ Puerta \\
\hline 10,2 & 8,1 & 3,3 & $6^{\circ}$ Puerta \\
\hline $11,2(2)$ & $10,2(2)$ & 4,2 & $7^{\circ}$ Puerta \\
\hline 12,Int. & 10,3 & $5^{\circ}$ Puerta $\underline{30}$ & $9^{\circ}$ Puerta \\
\hline 12,1 & 11,2 & $8^{\circ}$ Puerta & $10^{\circ}$ Puerta \\
\hline \multirow[t]{3}{*}{$12,3(2)$} & 12, Int. & 8,3 & $11^{\circ}$ Puerta \\
\hline & $12,3(2)$ & 11,1 & 12,2 \\
\hline & & $11,2^{\underline{31}}$ & \\
\hline
\end{tabular}

\begin{tabular}{|c|c|c|c|}
\hline \multicolumn{3}{|c|}{ Libro de las Cavernas } & $\begin{array}{c}\text { Libro de la } \\
\text { Tierra }\end{array}$ \\
\hline kkw & kkw & kkw zmAw & kkw \\
\hline $1,1(5)$ & $6,1,2(2)$ & 1,2 & $\mathrm{~A}, 1,1(2)$ \\
\hline $1,3(4)$ & $6,1,3$ & 1,5 & $\mathrm{~A}, 1,2(2)$ \\
\hline $1,4(2)$ & $6,1,4(2)$ & $3,3,2$ & $\mathrm{~A}, 1,3(2)$ \\
\hline $2,1(4)$ & $6,1,2$ & $3,3,5$ & $\mathrm{~A}, 2,2(1)^{\underline{32}}$ \\
\hline $2,2(2)$ & $6,2,2$ & $4,1,3$ & $\mathrm{~A}, 2,3(1)^{\underline{33}}$ \\
\hline $3,1,1$ & $6,2,3$ & $4,2,1$ & $\mathrm{~A}, 2,4(2)$ \\
\hline
\end{tabular}




\begin{tabular}{|c|c|c|c|}
\hline 3,1,3 (3) & $6,2,4$ & $4,3,2$ & A,3,2 \\
\hline $3,1,5$ & $6,2,5$ & 4, Int. (2) & $\mathrm{B}, 2(3)$ \\
\hline $3,2,1$ & $6,3,1$ & 4,3,3 & $\mathrm{B}, 5(1)^{\frac{34}{4}}$ \\
\hline $3,2,3(2)$ & $6,3,2$ & $5,3,1$ & C,1 (2) \\
\hline $3,2,5(2)$ & $6,3,4$ & $\begin{array}{c}\text { Escena de Osiris } \\
\text { (2) }\end{array}$ & C,3 \\
\hline $3,3,1$ & & & $\mathrm{C}, 5$ \\
\hline 3,3,3 (2) & & & C,7 (1) \\
\hline 4 Int. (17) & & & $\mathrm{D}, 1,1$ \\
\hline 5, Int. (15) & & & $\mathrm{D}, 2,2$ \\
\hline Escena de Nut (4) & & & $\mathrm{D}, 2,4$ \\
\hline $5,1,1(2)$ & & & $\mathrm{D}, 2,6$ \\
\hline $5,1,2$ & & & $\mathrm{D}, 2,7$ \\
\hline $5,2,1$ & & & $\mathrm{D}, 3,2$ \\
\hline $5,2,2$ & & & $\mathrm{D}, 3,3(3)$ \\
\hline $5,3,1$ & & & \\
\hline \multicolumn{4}{|l|}{ Escena de Osiris } \\
\hline \multicolumn{4}{|l|}{$5,4,1(2)$} \\
\hline \multicolumn{4}{|l|}{$5,5,1$} \\
\hline \multicolumn{4}{|l|}{ Prec 6 Int. (2) } \\
\hline \multicolumn{4}{|l|}{ Prec 6 1,1 (2) } \\
\hline \multicolumn{4}{|l|}{ Prec 6 Int. (2) } \\
\hline \multicolumn{4}{|l|}{ Prec 6 1,1 (2) } \\
\hline \multicolumn{4}{|l|}{ Prec 6 1,2 (2) } \\
\hline \multicolumn{4}{|l|}{ Prec 6, 3 (6) } \\
\hline \multicolumn{4}{|l|}{ Prec 6 5,1 } \\
\hline 61,1 & & & \\
\hline
\end{tabular}

La marcada diferencia entre el número de alusiones a kkw y a kkw-zmAw en los textos analizados, confirman la mayor diversidad de acciones que se producían en la primera en relación con la segunda. En este sentido, queda de manifiesto la omnipresencia de kkw como espacio permanentemente superado o anulado provisoriamente por el paso del disco, mientras que kkw-zmAw tiene una vinculación con un estado permanente, probablemente por fuera del mundo ordenado y donde los rayos de sol no tenían acceso.

\section{Conclusiones}

Creo que debemos ser flexibles a la hora de definir lo que cada uno de los términos abarca y explica. Ambos remiten a una realidad de falta de luz; provisoria -kkw- si tenemos en cuenta la dinámica de la presencia del disco en la Duat, y definitiva -kkw-zmAw- si nos atenemos a lo que Hornung propone sobre las áreas de aniquilación -Htmjt-.

No sólo Hornung, sino también Barta (véase cita 42) y Wilson $\frac{36}{}$ coinciden en afirmar una ubicación por fuera del mundo ordenado para kkw-zmAw. Ello supondría o significaría la presencia del desorden en el orden que Ra debe transitar cada noche, o bien, como afirman Barta (supra cita 40) y Hornung $\frac{37}{}$ habría que identificar a la Duat con kkw-zmAw, sobre todo teniendo en cuenta que en el Libro del Amduat, hora 12, 
segundo registro, kkw-zmAw y con frecuencia la Dat

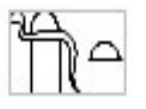

"profundidad", lleva frecuentemente el determinativo "Agua” y ello también en tiempos romanos (Hornung, 1956a:31) (específicamente en el papiro Carlsberg) $\underline{38}$.

No hay ninguna duda respecto a que la oscuridad es el rasgo distintivo de la Duat. Tanto kkw, kkw-zmAw como grH y snk pueden constituir su contexto general. En todo caso, si hubiera variantes, y efectivamente que las hay, no tienen que ver éstas con una ubicación exógena sino con el carácter hiperbólico para kkwzmAw que mencionamos anteriormente, y con un espacio específico, diferenciado del resto, en el que se ajusticia permanentemente y en el que se expulsa a los condenados $\underline{39}$, en el que el difunto sufre la tan temida segunda muerte o disolución absoluta, pasando así a formar parte de los $n \operatorname{xpr}^{40}$ o los no-existentes -tmw jwtyw- (Libro del Amduat, 3,3).

Cuando en la última hora del Libro del Amduat se dice que "la majestad de este Gran Dios (Ra) descansa en esta caverna en el final de kkw-zmAw”, la definición de "tinieblas de conexión” es la que más hace justicia al momento, dado que estamos en el punto de contacto entre el Más Allá y el Más Acá, en el que se marca el límite para Osiris -sSmw n wsjr "Imagen de Osiris" y sSmw-jwf "Imagen de Carne" (Barta, 1994:59). Permanece éste en la Duat- y por tanto en los primeros momentos en que la oscuridad es dejada atrás. Así, kkw-zmAw es vista aquí como el entorno de la Duat (en el sentido del texto cosmológico L del Papiro Carlsberg citado en p. 3), mientras que kkw se constriñe a la oscuridad hendida permanentemente por el disco. No obstante ello, ambas acepciones son partes constitutivas de la Duat.

En este sentido, la definición de Schott de kkw-zmAw como oscuridad "variada” o "indeterminada" (Schott, 1958:341) en la traducción demótica (papiro Carlsberg) $\frac{41}{}$, al hacer un breve recorrido diacrónico por la significación del término, es la más apropiada para la variedad procesual y contextual que proponemos en este estudio.

En todas las acciones que se dan en un contexto de oscuridad, kkw y kkw-zmAw son utilizadas en los textos en forma indistinta; es decir, y tomando ejemplos del Libro del Amduat en los que en la misma hora aparecen ambas acepciones:

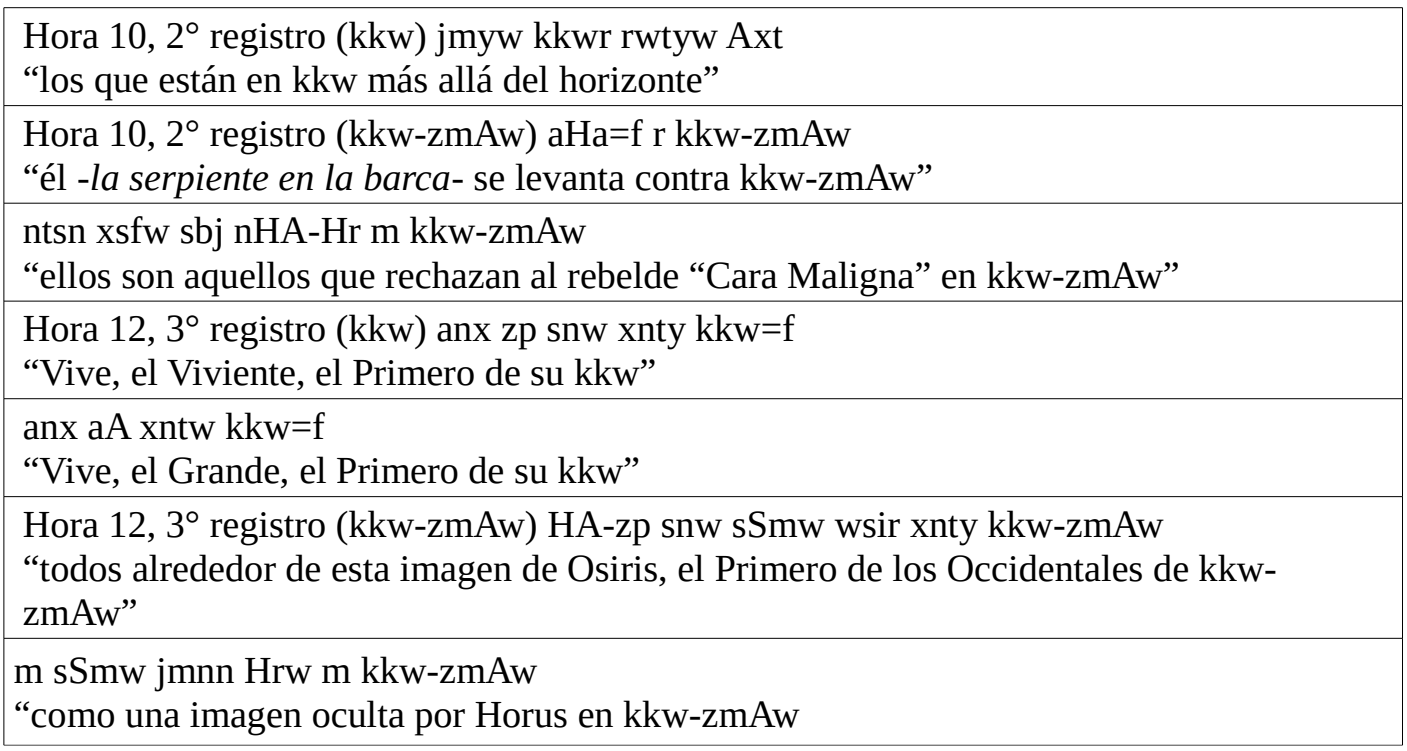


Es importante marcar que tanto en las acciones en que el sol "deja atrás” a la oscuridad, en las que "pasa y se

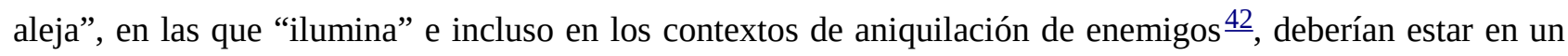
área por fuera del mundo creado $\frac{43}{3}$ siguiendo el análisis de Hornung. Los textos funerarios analizados contradicen esta aseveración, aunque es cierto que podría explicarse como "intromisiones" del caos en el orden, según su argumentación.

La diferencia entre la fuente cosmológica y la fuente funeraria es la diferencia entre el caos y el mundo ordenado. Entendemos que en este caso, un análisis "lineal” entre una fuente cosmológica (p. Carslberg) y una fuente funeraria como la aquí estudiada presenta incompatibilidades y en el peor de los casos, contradicciones.

Ambas acepciones son intercambiables y las fuentes nada dicen respecto de su locus exógeno al mundo creado. En la literatura de la Duat, entonces hay varias maneras de nombrar a la oscuridad en tanto falta o ausencia de luz (según la dinámica de la Duat) y kkw como kkw-zmAw no escapan a esta lógica.

De este modo, y aceptando entonces que nominalmente no hay diferencias entre kkw y kkw-zmAw en el contexto particular de los libros funerarios del Reino Nuevo, queda para una próxima discusión la cuestión de si ésta última está por fuera de la Duat, o si ambos conceptos son intercambiables o si incluso la Duat, al igual que kkw-zmAw está por fuera del mundo ordenado. Entendemos que, si bien hemos adelantado algunas conclusiones respecto de estas problemáticas, es conveniente dejar planteadas cuestiones que hacen al contexto más general de la concepción cosmológica y desde aquí ver cómo se opera en el espacio funerario particular. En todo caso, y aceptando los permanentes embates del caos en el orden, según la hipótesis

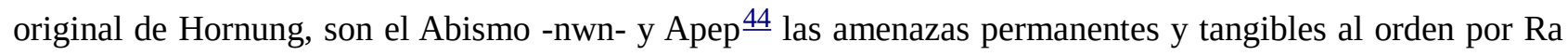
establecido.

\section{Notas}

1 Quiero agradecer la ardua tarea de los evaluadores quienes con sus invalorables aportes enriquecieron este artículo.

$\underline{2}$ “....antes de la creación existía un espacio sin límites, un espacio acuoso de aguas inertes, totalmente inmóviles rodeado de una absoluta oscuridad”, (Meeks, 1995:30).

3 Según Conman, “en Egipto no había Más Allá. La falsa idea de un Más Allá egipcio engendró otras ideas erróneas. Una idea equivocada que es casi universalmente sostenida por los egiptólogos es que el rey fallecido en los Textos de las Pirámides deseaba unirse a las estrellas circumpolares”, (Conman, 2003:37), idea que complementa con la confirmada "falta de evidencia para sostener cualquier idea egipcia del Más Allá”, (Conman, 2003:42). Para concluir, el autor reclama que "ningún Texto de las Pirámides especifica alguna localización para la dwAt, aunque otros la mencionan principalmente en el cielo; sin embargo ningún texto coloca a la dwAt bajo la tierra. Además, una descripción explícita dada por el papiro Carlsberg no coloca a la dwAt bajo la tierra”, (Conman, 2003:42-43). Obviamente que aceptar esta hipótesis es autoconspirar con lo que intentamos proponer en este artículo. Hacemos la mención dado lo novedoso de dicha hipótesis y porque el autor trabaja en profundidad cuestiones vinculadas a la arqueo-astronomía, a los mapas estelares, al nacimiento y derrotero de las estrellas y al hecho de señalar que "el tiempo que el sol está en la dwAt probablemente era un tiempo de poder mágico especial para las estrellas”, (Conman, 2003:61). Todas las traducciones de citas de otras lenguas corresponden al autor del presente artículo.

4 Para Hornung (1990:91-93) su datación corresponde a la Dinastía XIX; Allen (1988:1) propone su datación en el Reino Medio, mientras que Von Lieven (2007:223-254), principalmente en base a argumentos de carácter lingüístico, retrotrae la datación no sólo al Reino Medio sino incluso al Reino Antiguo. 
$\underline{5}$ “Su (Cuerpo de Nut) divide el Cosmos, creado en el mismo comienzo (zp tpy) por Ra-Atum de acuerdo a las reglas de la armonía universal, de lo amorfo, lo acromático, lo oscuro y la materia primigenia infinita, que se funde con lo que debería ser equivalente a la no-existencia y la catástrofe”, (Maravelia, 2003:63).

6 6 Denominado texto L del Papiro Carlsberg. Ver (Allen, 1988:9); (Clagett, 1995:374); (Barta, 1994: 177179); (Lull, 2006:46-47); (Von Lieven, 2007:59-60).

$\underline{7}$ Allen (1989:4) concluye que "oscuridad uniforme” es un término más "descriptivo que denotativo", por ello lo asimila a a nw, "Aguas” o “Aguas Primordiales”.

$\underline{8}$ jw nTrw jmjw pt / jw nTrw jmjw tA / jw nTrw jmjw dAt, "los dioses que están en el cielo / los dioses que están en la tierra / los dioses que están en la Duat”, Urk. IV 1192 9-11. Ocasionalmente pt - tA - nwn y pt - tA - kkw smAw, (Hornung, 1956a:50); también (Janák, 2011:122).

$\underline{9}$ "Relieve que describe al cielo, como la diosa Nut, extendida, como una dosel sobre la tierra (aquí calificada "arenas" y separada de ella por la atmósfera, en la forma del dios Shu. A lo largo del cuerpo del cielo, son descritas estrellas y el sol en varios estadios de su viaje diario. Los textos que acompañan explican ambas ilustraciones y sus relaciones con el universo visible y conjetural”, (Allen, 1988:1).

10 En el caso de los condenados, "sacrificados al término de su tiempo" (Libro de las Puertas 4,1), o de la “duración de la vida (...) para los bAw del Oeste” (Libro de las Puertas 5,3), las limitaciones impuestas por su condición, les evitaba dicha secuencialidad. El modo de citar los Libro del Más Allá del Reino Nuevo egipcio se hace de la siguiente manera: 1) “Libro”, 2) Hora, puerta, división, parte; 3) Registro y 4) Escena (según corresponda).

11 "Las descripciones de áreas (o espacios) más allá del cuerpo de la diosa Nut son representativos de la noción egipcia de "aquello lo cual no existe”. Incluso donde no hay nada, hay materializaciones de vacíos y habitantes del vacío. Aquí, principalmente en la región triangular sobre los brazos de Nut y también en el texto L que forma el borde superior de la viñeta, vemos que esta región está caracterizada no solamente con la frialdad y oscuridad que asociamos con el espacio, sino también con la humedad y lo pantanoso de lo frío y húmedo, lugares oscuros donde el sol nunca ilumina”, (Symons, 1999:163).

12 "La diversidad de estos libros del Reino Nuevo, explica la ostensible superpoblación y ocupación del espacio funerario, (...) contraído en uno sólo -Duat-, en una escala desconocida en las compilaciones anteriores. Asimismo, la variedad de habitantes supone no sólo una ampliación espacial, sino también, una diversidad procesual hasta entonces inexistente (situaciones vinculadas a realidades de transfiguración y/o eliminación)”, (Bonanno, 2014:87).

13 "Los elementos que constituyen el estado de cosas anterior a la creación -océano primordial, tinieblas (cursiva agregada), pereza, negación- están doblemente presentes en el mundo creado. Por un lado, como último límite o como ámbito más allá de todo límite con el que uno se encuentra cuando uno sobrepasa la limitación de lo existente; por otro lado, dentro de la creación ordenada, en medio de nosotros mismos”, (Hornung, 1999:164).

14 Hay regiones especiales, sobre todo aquellas en que se castiga a los condenados -xftyw- (principalmente en los registros inferiores del Libro de las Cavernas), o también en los caminos de Rosetau de la $4^{\circ}$ hora o la jmHt de la $5^{\circ}$ hora, ambas del Libro del Amduat.

15 Ello se plasma en términos onto-teológicos, en debilidad re-conversión (intercambio) apoteosis. Este correlato se explica por el efecto del disco sobre los cuerpos; la oscuridad es el marco de la debilidad y la necesidad de contar con el paso del disco; luego, su presencia (del disco) opera una reconversión en estos cuerpos debilitados, fortificándolos en todo sentido, para finalmente con la nueva lejanía que el paso supone, 
los cuerpos reconvertidos retornan a la quietud/oscuridad original pero ahora renovados.

16 En Libro del Amduat, hora 12,Int., "la majestad de este gran dios descansa "en esta caverna del final de la oscuridad absoluta” -orrt njt pHwy kkw-zmAw- (Barta, 1985:178).

17 Para Barta (1985:179, nota 735), son las tinieblas primordiales las que pueden entenderse como expresión sinónima del Más Allá -Unterwelt-”.

$\underline{18}$ Urk. IV 248, 16-18. Transliteración de Hornung (1956b:30). La traducción es propia.

19 “Vereinigende Finsternis” es la traducción de Sethe (1928:5).

20 Para Hornung (1956a:49) “en las guías del Más Allá del Reino Nuevo, las tinieblas son inseparables de la Duat” y es evidente que tinieblas primordiales “-Urfinsternis- es usado como sinónimo para la Duat, no como una categoría particular”, (Hornung, 1956a:50).

$\underline{21}$ Una mención especial a oscuridad alude a los "guardianes de la gran oscuridad” - aAtyw kkw - (la Duat) en el Libro de las Cavernas 1,5, (Piankoff, 1942: lámina 8).

$\underline{22}$ "[L]a oscuridad les envuelve de nuevo cuando les ha pasado" (3,1,1), "yo (disipo) la oscuridad para el Rey Rameses VI por mi gran disco con rayos" $(3,1,3)$, "Estos dioses ven los rayos de su disco detrás de él y cuando él les ha pasado la oscuridad les envuelve” (3,1,3), “yo paso iluminándolos (a los dioses) y disipando su oscuridad" (3,1,5), "La oscuridad les cubre después de que este Gran Dios ha pasado cerca de ellos" $(3,2,3)$

$\underline{23}$ “La salida que hace el Gran Dios de la oscuridad absoluta en la Caverna de Osiris”.

$\underline{24}$ “Este Gran Dios sale de la oscuridad en la Caverna de Osiris”.

$\underline{25} \mathrm{O}$ bien un contexto de una particular y marcada pasividad -objetivado luego- como en el de la hora ocho del Libro del Amduat en el que la puerta que comunica al primero con el segundo registro se denomina Ds zmAw kkw, "cuchillo de la oscuridad unificada".

$\underline{26}$ Así por ejemplo, “que su (habitantes del registro) oscuridad -kkw- sea iluminada”, "la oscuridad -kkw- de la tierra es iluminada”, (Libro del Amduat 6,1); "su oscuridad -kkw- es iluminada, (Libro del Amduat 6,3); “atravieso los misterios de la Duat, mi disco ha entrado en el lugar misterioso, para alumbrar a los que están en su oscuridad -kkw-, (Libro de las Cavernas 4,1,1); Estos dioses que no ven la luz de Ra, (pero) vigilan mientras permanecen en su oscuridad -kkw=f-, respiran cuando escuchan su voz, sus bAw pasan después de él, (Libro de la Tierra A,1,3); “abran sus capillas para que mis rayos puedan penetrar en su oscuridad -kkw-”, (Libro de las Puertas 3,1).

$\underline{27}$ “los que están en su Desierto se lamentan al escuchar el cierre de esta puerta” (Libro de las Puertas, 2, Int.).

$\underline{28}$ Un problema similar plantea el espacio denominado Htmjt, “zona de neutralización y/o aniquilación”, casi omnipresente sobre todo en los niveles inferiores del Libro de las Cavernas, (infra cita 68). Hornung (1999a: 164) concluye que es "el límite insuperable en el cual se acaba la influencia de los dioses y del rey", por lo que "en estas regiones infernales del Mundo Inferior egipcio no se trata de torturar a los pecadores condenados sino de borrar su existencia en aquel ominoso "lugar de destrucción” (Htmjt), Hornung (1999:168). Para Barta (1985:183) "ese lugar [Htmjt] está en las tinieblas primordiales [cursivas añadidas], es decir que no pertenece al Más Allá -Unterwelt-, sino que hay que buscarlo afuera del cosmos ordenado”. Como vemos, del mismo modo que con $k k w$ zmAw, el espacio Htmjt presenta controversias similares en cuanto a su locación, aunque si tenemos en cuenta los textos en los que aparece -literatura funeraria- y que para quienes la habitan Ra les dice que "ustedes no verán mis rayos y no tendrán más poder contra mis 
rayos" (Libro de las Cavernas 3,3,2), podemos sostener la segunda afirmación de Hornung y concluir que, siendo parte de la Duat, estamos en presencia de los lugares más profundos, oscuros, que el disco evita, pero que no dejan de pertenecer al mismo espacio Duat, por lo tanto, espacio ordenado.

$\underline{29}$ Llamamos espiritualización a aquellos efectos derivados tanto de la influencia de cualquier entidad divina que sobre el difunto opera una re-significación de sus signos pneumáticos, a posteriori a su regeneración física como de los efectos derivados de la actividad ritual. Así por ejemplo y puntualmente en la literatura que estamos trabajando, $\mathrm{nt}=\mathrm{Tn} \mathrm{nn} \mathrm{n}$ nTrwt a bAyt -"son estas diosas que conceden la espiritualidad” (Libro de las Cavernas Prec 6,2,2), o jr tmw n ra sAxw nTr dwA bA=f r aat dmdw m xftjw=f -"lo que Atum hace por Ra: (él) espiritualiza al dios, adora su bA, causa el mal a sus enemigos”- (Libro de las Puertas 1,2). Previamente, en los Textos de los Sarcófagos -TdS-, hay referencias a esta realidad; así por ejemplo con motivo de "la apertura de los portales del cielo y la cesión del trono de Geb” (TdS 487 VI 66a-r), -dominio sobre los dos ámbitos-, la cesión del cielo y el brillo, haciéndolo un Ax con poder sobre los vivos (TdS 722 VI 350 r-s 351a-b) -brillo como esencia divina y transfiguración- y en general aquellas declaraciones que confirman el poder recompuesto de N. sobre la extra-corporeidad (TdS $412 \mathrm{~V}$ 239a-c) -anámnesis en el cielo-, (882 VII 93c) “-atravesar el crepúsculo de Ra-, -existir en la plenitud de Osiris-” (TdS 767 VI 399ac), sólo para mencionar una parte ínfima. Es necesario retener la secuencia diacrónica cuerpo (Dt)-Ax.

$\underline{30}$ En negrita se indica que en esas puertas en particular la grafía es tanto kkw como kkw-zmAw.

$\underline{31}$ Snk

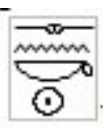
también aparece en el segundo registro de la hora once; ver Wb IV 175-176 (1971).

$\underline{32}$ En dos ocasiones, grH.

33 En una ocasión, grH.

34 Por HAp Hr=sn <kkw>, (Piankoff, 1953:31).

35 Por HAp Hr=sn <kkw>. La última palabra en escritura enigmática, (Piankoff, 1953: lámina 20).

36 "la oscuridad más allá del horizonte, antes de la creación y fuera del mundo creado es llamada kkwzmAw unida, esto es, “oscuridad intensa”, (Wilson, 1997:1091-1092).

$\underline{37}$ "El reino de los muertos está fuera de la creación (...) kkw-zmAw, el Nun y las cavernas de los demonios del Más Allá se extienden en lo ilimitado, en el "no-Ser” [tm wnn], a los que los enemigos del dios Atum transfieren“, (Hornung, 1956a:30).

$\underline{38}$ Agua y Oscuridad son dos elementos presentes en el mito heliopolitano de la creación. El caos acuoso de la pre-creación era precisamente una oscura inmensidad de agua sumida en la más profunda oscuridad.

$\underline{39}$ “...que rodea lo espacial como temporal de la totalidad de la creación, y a la Duat Inferior y Superior”, (Hornung, 1968:14).

40 Así en el Libro del Amduat 11,3

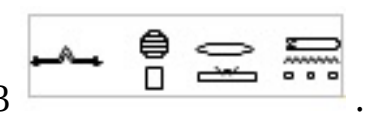

$\underline{41}$ "Finsternis welche vermischt ist”, es la traducción de kkw-zmAwcomo kkw iw.f Sbn tal como aparece en el Papiro Carlsberg, (Hornung, 1956a:33).

42 Hmtyw wnnyw kkw.sn, "ustedes son los aniquilados en kkw” (Libro de las Cavernas 4,3,2). Htm xtyw xnty dwAt kky xfty n tyw HD=sn nn xftyw nw Wsjr XrwsDt ssw sDtw nt=sn "Aniquilados están los enemigos de El que está a la Cabeza de la Duat, que están en la oscuridad, que no tienen luz, estos enemigos de Osiris que están en la llama, con sus cuerpos quemados. (Libro de las Cavernas Prec. 6,3). 
43 “...la absoluta oscuridad debe por eso ser entendida como caótica, situada fuera de la creación, distante del mundo, a la que la luz del sol nunca ilumina”, (Barta, 1985:178). En el mismo sentido, Greyer, (1993:109) concluye que kkw-zmAw indica un límite absoluto en el Más Allá que está desconectado con una dirección geográfica.

$\underline{44}$ “Apep, su voz rodea la Duat”, Libro de las Puertas 11,1,

\section{Bibliografía}

Abt, Th.; Hornung, E, (2007). The Egyptian Amduat. The Book of the Hidden Chamber. Zurich: Living Human Heritage Publications.

Allen, J. P. (1988). Genesis in Egypt: The Philosophy of Ancient Egyptian Creation Accounts. Yale Egyptological Studies, 2: New Haven.

Barta, W. (1985). Die Bedeutung der Jenseitsbücher für den vestorbenen König. MunchenerMunchner Ägyptologische Studien 42. Munich: Deutscher Kunstverlag.

Barta, W. (1994). Komparative Untersuchungen zu vier Unterweltsbüchern. Frankfurt am Main - Bern - New York - Paris: Peter Lang.

Bonanno, M. (2014). La Duat como espacio de una dialéctica de la regeneración. Definiciones acerca del vínculo Ra-Osiris en los Textos del Amduat. In-habitación y resignificación del espacio funerario. Tesis para optar por el grado de Doctor en Historia. Universidad Nacional de La Plata. Recuperado de http://sedici.unlp.edu.ar/handle/10915/39679.

Brunner, H. (1954-1955). Die Grenzen von Zeit und Raum bei den Äegypten, Archiv für Orientforschung 1: Universität Wien: Wien.

Conman, J. (2003). It`s about Time: Ancient Egyptian Cosmology, Studien zur Altägyptischen Kultur, 31, 3371.

De Buck, A. (1954). The Egyptian Coffin Texts. Vol 5. Oriental Institute Publications 73: Chicago. .

De Buck, A. (1954). The Egyptian Coffin Texts. Vol 5. Oriental Institute Publications 73: Chicago. .

De Buck, A. (1956). The Egyptian Coffin Texts. Vol 6. Oriental Institute Publications 81: Chicago.

De Buck, A. (1961). The Egyptian Coffin Texts. Vol 7. Oriental Institute Publications 87: Chicago.

Greyer, J. (1993). Ezequiel 27 and the Cosmic Ship. En Davies P. Clines D. (Eds.). Among the Prophets. Language, Image and Structure in the Prophetic Writings (pp. 105-126). England: Sheffield Academic.

Erman A.; Grapow, H. (1971). Wörterbuch der Ägyptischen Sprache. Vols. 4 y 5 Berlin: Akademie Verlag.

Frankfort, H. (1933). The Cenotaph of Seti I in Abidos. 2 Vols. Egypt Exploration Society 36 y 39. London: Offices of the Egypt Exploration Society.

Hegenbarth-Reichardt, I. (2009). Von Zeiten und Räumen. Oder: Wie unendlich ist die altägyptische Ewigkeit?. En Kratz R. y Spieckerman H. (Eds.) Zeit und Ewigkeit als Raum göttlichen Handelns (pp. 3-28). Berlin: Walter De Gruyter \& Co.

Hornung, E. (1956a). Nacht und Finsternis im Weltbild der Alten Ägygpten.Inaugural-Dissertation zur Erlangung des Doktorgraden einer Hohen Philosophischen Fakultät der Eberhard Karl Universität zu 
Tübingen, Tübingen.

Hornung, E. (1956b). Chaotische Bereiche in der geordneten Welt, Zeitschrift für ägyptische Sprache und Altertumskunde 81, 28-32.

Hornung, E. (1963). Das Amduat. Die Schrift des verborgenen Raumes., Ägyptologische Abhandlungen 7, 2 Vols. O. Harrassowitz: Wiesbaden.

Hornung, E. (1967). Das Amduat. Die Schrift des verborgenen Raumes. Die Kurzfassung. Nachträge. O. Harrassowitz: Wiesbaden.

Hornung, E. (1968). Altägyptische Höllenvorstellungen. Berlin: Akademie Verlag.

Hornung, E. (1974). ”Dat”. Lexikon der Ägyptologie I, cols. 994-995. O. Harrasowitz: Wiesbaden.

Hornung, E. (1975). ”Dunkelheit”. Lexikon der Ägyptologie I, cols. 1153-1154. O. Harrasowitz: Wiesbaden.

Hornung, E. (1987-1994). Texte zum Amduat. Aegyptiaca Helvetica 13-15, Genève: Editions de Belles Lettres.

Hornung, E. (1979-1980). Das Buch von den Pforten des Jenseits. Aegyptiaca Helvetica 7-8, Basel, Genève: Editions de Belles Lettres.

Hornung, E. (1990). Zwei ramessidische Könisgraber: Rames IV und Ramses VII. En Assmann J. (Ed.) Theben (pp. 7-21) Mainz: von Zabern.

Hornung, E. (1999a) El Uno y los Múltiples. Concepciones egipcias de la divinidad. Ed. Trotta: Madrid.

Hornung, E. (1999b). The Ancient Egyptian Books of the Afterlife. Ithaca: Cornell University Press.

Janák, J. (2011). The Structure of the Egyptian Pantheon. En Vacín L. (Ed.). Ancient Near Eastern Studies in Memory of Blahoslav Hruška (pp. 119-127). Dresden: Islet Verlag.

Lull, J. (2006). La astronomía en el antiguo Egipto. Valencia: Universidad de Valencia.

Manassa, C. (2006). The Judgment Hall of Osiris in the Book of Gates, Revue d'Egyptologie 57, 109-141.

Maravelia A. (2003). Cosmic Space and Archetypal Time: Depictions of the Sky-Goddess Nut in three royal tombs of the New Kingdom and her relation to the Milky Way, Göttinger Miszellen, 197, 55-72.

Marshall, C. (1995). Ancient Egyptian Science: Vol. 2 Calendars, clocks and Astronomy. Philadelphia: American Philosophical Society.

Maystre, Ch.; Piankoff, A. (1939-1962). Le Livre des Portes. 3 vols. Le Caire. Mémoires publiés par les membres de l’Institut Français `Archéologie Orientale.

Meeks, D.; Favard-Meeks, Ch. (1995). La vida cotidiana de los dioses egipcios. Madrid: Ed. Hachette.

Morenz, S. (1992). Egyptian Religion. Ithaca: Cornell University Press.

Murray, M. (1904). The Osireion at Abydos. London: Gilbert and Rivington LTD.

Neugebauer, O.; Parker, R. (1960). Egyptian Astronomical Texts I, London: Brown University Press.

Parker, R. (1974). Ancient Egyptian Astronomy. Philosophical Transactions of the Royal Society of London. Serie A, Mathematical, Physical and Engineering Sciences, 276 (1257), 51-65.

Piankoff, A. (1942). "Le livre des Quererts. 1er tableau”, Bulletin de l'Institut français d'archéologie orientale 41, 1-11. 
Piankoff, A. (1944). "Le livre des Quererts. Seconde division - Cinquième division”, Bulletin de l'Institut français d'archéologie orientale 42, 1-62.

Piankoff, A. (1945). “Le Livre des Quererts Sixième division: fin du livre des Quererts”, Bulletin de l'Institut français d'archéologie orientale 43, 1-50.

Piankoff, A. (1953). La Creation du disque Solaire. Le Caire. Biblothèque d`Ètude Institut français d 'archéologie orientale 19.

Piankoff, A. (1964). The Litany of Re. Egyptians Texts and representations, New York: Pantheon Books.

Roberson, J. (2007). An Enigmatic Wall from the Cenotaph of Seti I at Abydos, Journal of the American Research Center in Egypt, 43, 93-112.

Schott, S. (1958). Die Schrift der verborgenen Kammer in Königsgräbern der 18. Dynastie. Nachrichten von der Akademie der Wissenschaften zu Göttingen 4. Göttingen: Vandenhoeck \& Ruprecht.

Sethe, K. (1906-1909). Urkunden der 18. Dynastie, historisch-biographische Urkunden, 4 Vols. Leipzig: Hinrichs.

Sethe, K. (1928). Altägyptische Vorstellungen vom Lauf der Sonne. Berlin: De Gruyter.

Symons, S. (1999). Ancient Egyptian Astronomy: Timekeeping and Cosmography in the New Kingdom. Thesis submitted for the Degree of Doctor of Philosophy Department of Mathematics and Computer Science, University of Leicester, Leicester.

Von Lieven, A. (2007). Grundriss des Laufes der Sterne: das sogenannte Nutbuch, Volumen 1 CNI Publications, Copenhagen: Museum Tusculanum Press.

Von Lieven, A. (2010). Translating the Fundamental of the Course of the Stars. En Imhausen A. y Pommerening T. (Eds.).Writings on Early Scholars in the Ancient Near East, Egypt, Rome, and Greece (pp. 139-150). Berlin/New York: De Gruyter.

Wiebach-Koepke, S. (1997). "Standorte - Bewegungstypen - Kreisläufe Semantische Betrachtungen zur Dynamik der Sonnenlaufprozesse in Amduat und Pfortenbuch”, Studien zur Altägyptischen Kultur, 24, 337366.

Wiebach-Koepke, S. (2007). Sonnenlauf und Kosmiche Regeneration. Zur Systematik der Lebensprozesse in den Unterweltsbüchern. Wiesbaden: Harrassowitz Verlag.

Wilson, P. (1997). A Ptolemaic Lexikon. A Lexicographical Study of the Texts in the Temple of Edfu. Orientalia Lovaniensia Analecta 78.Leuven: Peeters. 